\title{
Circulant matching method for multiplexing ATM traffic applied to video sources *
}

\author{
K. Spaey C. Blondia \\ University of Antwerp \\ Department of Mathematics and Computer Science \\ Performance Analysis of Telecommunication Systems Research Group \\ Universiteitsplein 1 \\ B-2610 Wilrijk - Belgium \\ \{spaey,blondia\}@uia.ua.ac.be
}

\begin{abstract}
In this paper a method is proposed, called circulant matching method, to approximate the superposition of a number of discrete-time batch Markovian arrival sources by a circulant batch Markovian process, while matching the stationary cumulative distribution and the autocorrelation sequence of the input rate process. Special attention is paid to periodic sources. The method is applied to the superposition of MPEG video sources and the obtained results are validated through experiments.
\end{abstract}

\section{Keywords}

Multiplexing, circulant matching method, periodic sources

\section{INTRODUCTION}

Defining appropriate models for traffic streams in ATM networks has been the subject of intensive research during the past ten years. Several models, together with the corresponding queueing systems, have been proposed. In this paper, a matrix-analytical approach is followed, by choosing discete-time batch Markovian arrival processes (D-BMAPs) (see (Blondia 1993)) as model for ATM traffic. A basic problem in traffic engineering of ATM networks is the computation of the buffer occupancy and waiting time distribution of a single server queue with deterministic service time (i.e. the time needed to transmit a cell) and input consisting of a superposition of processes modeling ATM traffic streams. A major problem encountered when solving this system

*This work was supported by the Commission of the European Union, under the project ACTS AC094 EXPERT 
is the explosion of the state space when the number of input sources takes values that are typical for real life situations.

In this paper, a solution is proposed which reduces the state space of this model drastically. This method, called circulant matching, is based on an approach proposed in (Hwang et al. 1995) and makes use of a traffic spectral representation, which was first introduced to queueing analysis by S.Q. Li et al. (see e.g. (Li et al. 1992)). A number of independent D-BMAPs form the input process of a single server queue. The exact superposition of these processes is again a D-BMAP, with number of states equal to the product of the number of states of the individual D-BMAPs. The idea is to replace this D-BMAP by a D-BMAP with a smaller circulant transition matrix which matches two important statistical functions of the exact input rate process, namely the stationary cumulative distribution and the autocorrelation sequence (characterized in the frequency domain by means of the power spectrum). The transition matrix is chosen to be circulant in order to avoid solving an inverse eigenvalue problem. Once the circulant D-BMAP is found, the problem is reduced to solving a $\mathrm{D}-\mathrm{BMAP} / \mathrm{D} / 1 / \mathrm{K}+1$ queue, for which efficient methods to compute the stationary queue length distribution are available (see e.g. (Blondia et al. 1992)).

The proposed approach is illustrated by means of the evaluation of the cell loss ratio (CLR) in a multiplexer that is fed by a number of video sources. The CLR values resulting from the circulant matching method are compared with those obtained from a series of experiments (Aarstad et al. 1998) performed within a European project in the ACTS programme, EXPERT.

The paper is organized as follows. Section 2 gives a detailed description of the circulant matching method, emphasising the differences with the approach proposed by S.Q. Li and paying special attention to periodic sources. In Section 3, the results are applied to the superposition of video sources, in particular to an MPEG source type model. Comparison with the experimental results is made in Section 4. Finally, conclusions are drawn in Section 5.

\section{MULTIPLEXING OF DISCRETE-TIME BATCH MARKOVIAN ARRIVAL PROCESSES}

\subsection{Discrete-time batch Markovian arrival process}

A class of stochastic processes which is often used to describe the stochastic nature of an ATM source is the class of processes which are modulated by a Markov chain. A discrete-time process which belongs to this class is a discretetime batch Markovian arrival process (D-BMAP) (Blondia 1993).

A D-BMAP is characterised by a sequence of matrices $\left(D_{k}\right)_{k \geq 0}$ and can be defined as follows: the matrix $D=\sum_{k=0}^{\infty} D_{k}$ is the transition matrix of a discrete-time Markov chain of dimension $m+1$. Suppose that at time $n$ this 
chain is in some state $i, 0 \leq i \leq m$. At the next time instant $n+1$, there occurs a transition to another state $j, 0 \leq j \leq m$ with probability $(D)_{i, j}$ at which a batch arrival may or may not occur. The matrix $D_{0}$ governs transitions that correspond to no arrivals while the matrices $D_{k}, k \geq 1$, govern transitions that correspond to arrivals of batches of size $k$. Let $\pi$ be the stationary distribution of $D$, i.e. $\pi D=\pi$ and $\pi \boldsymbol{e}=1$, where $\boldsymbol{e}$ is a column vector of 1's. The mean arrival rate $\lambda$ of the process is then given by $\lambda=\pi\left(\sum_{k=0}^{\infty} k D_{k}\right) e$. For more details and properties, see (Blondia 1993).

\subsection{Multiplexing of D-BMAPs: problem description}

The superposition of $M$ independent D-BMAPs $\left(D_{k}^{(i)}\right)_{k \geq 0}$, can again be described by a D-BMAP $\left(D_{k}\right)_{k \geq 0}$ with $D=\bigotimes_{i=1}^{M} D^{(i)}, D_{0}=\bigotimes_{i=1}^{M} D_{0}^{(i)}, D_{1}=$ $D_{1}^{(1)} \otimes\left(\otimes_{i=2}^{M} D_{0}^{(i)}\right)+\ldots+\left(\otimes_{i=1}^{M-1} D_{0}^{(i)}\right) \otimes D_{1}^{(M)}, \ldots$ where $\otimes$ denotes the Kronecker product. It is clear that this superposition leads to a state space explosion, which implies that in practice, $\left(\boldsymbol{D}_{k}\right)_{k \geq 0}$ is not usable. Therefore, the exact aggregate arrival process is replaced by another, but simpler process, which matches the exact one as close as possible for some important statistical functions. Preferable, the new process is again a D-BMAP, such that it is possible to keep on working within the same framework.

In (Hwang et al. 1995), it is proposed to replace the superposition of continuous-time Markov modulated Poisson processes (MMPPs) by a MMPP with a special structure, called circulant modulated Poisson process (CMPP). This process has a completely different Markovian structure from the superposition, but approximates two important statistical functions of the input rate process: the cumulative distribution representing the stationary statistics and the autocorrelation function in the time domain or equivalently the power spectral function in the frequency domain representing the second order statistics. In the next section it will be demonstrated that this method can be adapted for discrete-time Markov models such as D-BMAPs without too much difficulties, and leading to the result that the superposition of D-BMAPs can be replaced by a circulant D-BMAP. Simultaneously, the method will be extended such that the periodicity which is often noticed in the transition matrix of D-BMAPs, and thus also in their exact superposition, is preserved. Examples of periodic Markov sources are the MPEG model which will be used in section 3 and the model to describe the traffic profile of a tagged CBR connection after it has been jittered by background traffic (Blondia et al. 1995). Recent works studying and capturing periodicities present in real-time applications are e.g. (Lazar et al. 1993) and (Landry et al. 1997).

\subsection{The circulant matching method}

The input rate process of a D-BMAP $\left(D_{k}\right)_{k \geq 0}$ which is assumed to be irreducible and diagonalizable is defined by $\Gamma(m)$, with $\Gamma(m)=\Gamma_{i}$ while the 
Markov chain is in state $i$ at the $m$-th time slot. The input rate in a slot is thus a stochastic variable $\Gamma$ which takes the values $\Gamma_{0}, \ldots, \Gamma_{N-1}$ with probabilities $\pi_{0}, \ldots, \pi_{N-1}$, where

$$
\Gamma_{i}=\sum_{j=0}^{N-1}\left(\sum_{k=0}^{\infty} k D_{k}\right)_{i, j}=\left(\sum_{k=0}^{\infty} k D_{k} e\right)_{i} .
$$

The autocorrelation sequence $R[n]$ of the random process $\Gamma(m)$ is defined as $R[n]=E[\Gamma(m) \Gamma(m+n)]$. This gives for a D-BMAP

$$
\begin{aligned}
& R[0]=\pi\left[\left(\sum_{k=0}^{\infty} k D_{k} e\right) \odot\left(\sum_{k=0}^{\infty} k D_{k} e\right)\right], \\
& \underset{\substack{R \\
n \neq 0}}{R[0}=\pi\left(\sum_{k=0}^{\infty} k D_{k}\right) D^{|n|-1}\left(\sum_{k=0}^{\infty} k D_{k}\right) e,
\end{aligned}
$$

where $\odot$ denotes the element-by-element product of two vectors.

As $D$ is supposed to be diagonalizable, $D$ can be written as $D=\sum_{l=0}^{N-1} \lambda_{l} g_{l} h_{l}$, where $\lambda_{l}$ is the $l$-th eigenvalue of $\boldsymbol{D}$ and $\boldsymbol{g}_{l}$, resp. $\boldsymbol{h}_{\boldsymbol{l}}$, is the corresponding right column, resp. left row, eigenvector such that $\boldsymbol{h}_{l} \boldsymbol{g}_{l}=1$, from which

$$
\underset{n \neq 0}{R[n]}=\sum_{l=0}^{N-1}\left(\lambda_{l}\right)^{|n|-1} \psi_{l}, \quad \text { with } \quad \psi_{l}:=\pi\left(\sum_{k=0}^{\infty} k D_{k}\right) g_{l} h_{l}\left(\sum_{k=0}^{\infty} k D_{k}\right) e .
$$

Remark that if $\lambda_{l}=1, \sqrt{\psi_{l}}$ is the mean arrival rate.

Since $\boldsymbol{D}$ is the transition matrix of an irreducible Markov chain, $\left|\lambda_{l}\right| \leq 1$, $\forall l$, and 1 is always a simple eigenvalue of $\boldsymbol{D}$ which will be given the index zero: $\lambda_{0}:=1$. For each eigenvalue, denote $\lambda_{l}=\left|\lambda_{l}\right| e^{i \omega_{l}}$ and $\psi_{l}=\left|\psi_{l}\right| e^{i \theta_{l}}$ and define $\Omega$ to be the collection of all eigenvalues of $D: \Omega=\left\{\lambda_{0}, \ldots, \lambda_{N-1}\right\}$. By distinguishing between the different types of eigenvalues, from (3) one derives after some manipulation that

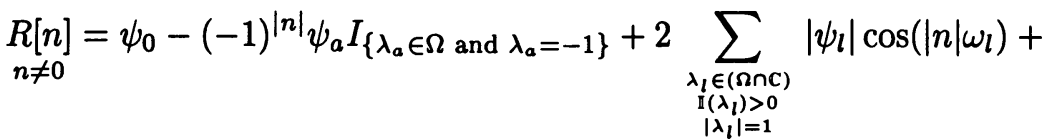

$$
\begin{aligned}
& \sum_{\lambda_{l} \in(\Omega \cap \mathbb{R} \backslash\{0,1,-1\})}\left(\lambda_{l}\right)^{|n|-1} \psi_{l}+2 \sum_{\substack{\lambda_{l} \in(\Omega \cap n) \\
l\left(\lambda_{l}|>0\\
| \lambda_{l} \mid<1\right.}}\left|\lambda_{l}\right|^{|n|-1}\left|\psi_{l}\right| \cos \left(|n| \omega_{l}-\omega_{l}+\theta_{l}\right),
\end{aligned}
$$

where $I_{\{a\}}$ is the indicator function of event $a$.

Remark that the second and fourth term in this expression disappear if the transition matrix $\boldsymbol{D}$ is aperiodic, since only periodicity of $\boldsymbol{D}$ implies the existence of eigenvalues different from 1 on the unit-circle.

The autocorrelation sequence in the time domain is equivalently characterized in the frequency domain by its power spectrum $P(\omega)=\sum_{k=-\infty}^{+\infty} R[k] e^{-i k \omega}$, which is the discrete-time Fourier transform of the autocorrelation sequence. 
Since $P(\omega)$ is periodic in $\omega$ with period $2 \pi$, only angular frequencies (expressed in $\mathrm{rad} / \mathrm{sec}$ ) in the range $-\pi<\omega \leq \pi$ need to be considered. By using (4), a formula for $P(\omega)$ is obtained from which the contribution of each eigenvalue of $\boldsymbol{D}$ to $P(\omega)$ can easily be read:

$$
\begin{aligned}
& P(\omega)=R[0]-\psi_{0}+2 \pi \psi_{0} \delta(\omega)+\psi_{a}-2 \pi \psi_{a} \delta(\omega-\pi) I_{\left\{\lambda_{a} \in \Omega \text { and } \lambda_{a}=-1\right\}} \\
& +2 \sum_{\lambda_{l} \in(\Omega \cap \mathbb{R} \backslash\{0,1,-1\})} \psi_{l} \frac{\cos \omega-\lambda_{l}}{1-2 \lambda_{l} \cos \omega+\left(\lambda_{l}\right)^{2}} \\
& +\sum_{\substack{\lambda_{l} \in(\Omega \cap n) \\
l\left(\lambda_{l}\right)>0 \\
\left|\lambda_{l}\right|=1}}\left(-2\left|\psi_{l}\right|+2 \pi\left|\psi_{l}\right| \delta\left(\omega+\omega_{l}\right)+2 \pi\left|\psi_{l}\right| \delta\left(\omega-\omega_{l}\right)\right) \\
& +4 \sum_{\substack{\lambda_{1} \in\left(\Omega ( n ) \\
\text { anc } \\
\left(\lambda_{l} l>0 \\
\left|\lambda_{l}\right|<1\right.\right.}} \mathbb{R}\left\{\psi_{l} \frac{\cos \omega-\lambda_{l}}{1+\left(\lambda_{l}\right)^{2}-2 \lambda_{l} \cos \omega}\right\} \quad \text { with }-\pi<\omega \leq \pi,
\end{aligned}
$$

where $\delta$ is the Dirac delta function.

As can be seen from this formula, the discrete part in the power spectrum is caused by the eigenvalues with modulus 1 .

If now not only one D-BMAP is considered, but $M$ independent D-BMAPs $\left(D_{k}^{(i)}\right)_{k \geq 0}, 1 \leq i \leq M$, the autocorrelation sequence of the superposition is given by

$$
R[n]=\sum_{i=1}^{M} R^{(i)}[n]+2 \sum_{i=1}^{M} \sum_{j=i+1}^{M} \sqrt{\psi_{0}^{(i)}} \sqrt{\psi_{0}^{(j)}}
$$

and thus

$$
P(\omega)=\sum_{i=1}^{M} P^{(i)}(\omega)+4 \pi \delta(\omega) \sum_{i=1}^{M} \sum_{j=i+1}^{M} \sqrt{\psi_{0}^{(i)}} \sqrt{\psi_{0}^{(j)}} .
$$

This means that the power spectrum of the aggregate process is completely known by all the eigenvalues of the D-BMAPs $\left(D_{k}^{(i)}\right)_{k \geq 0}$ and their contribution to their power spectrum. If now all $M$ D-BMAPs are identical, or can be divided into a limited number of groups of identical D-BMAPs, a lot of contributions to $P(\omega)$ come from the same eigenvalues and can be merged together. Thus, if a D-BMAP $\left(\boldsymbol{Q}_{k}\right)_{k \geq 0}$ could be constructed with as eigenvalues of $Q$ the eigenvalues that contribute to $P(\omega)$ in (7), a new D-BMAP could be obtained with the same power spectrum $P_{c}(\omega)$ provided that it is possible to tune the $\left(\psi_{c}\right)_{l}$ 's of this new D-BMAP.

To avoid the construction of $\boldsymbol{Q}$ to be equivalent to solving an inverse eigenvalue problem, $\boldsymbol{Q}$ must be such that its eigenvalues are known in closed form. 
As in (Hwang et al. 1995), a $N$-dimensional circulant is used:

$$
\boldsymbol{Q}=\left(\begin{array}{cccc}
a_{0} & a_{1} & \ldots & a_{N-1} \\
a_{N-1} & a_{0} & \ldots & a_{N-2} \\
\vdots & \vdots & \ddots & \vdots \\
a_{1} & a_{2} & \ldots & a_{0}
\end{array}\right)
$$

Its eigenvalues are given by $\left(\lambda_{c}\right)_{l}=\sum_{j=0}^{N-1} a_{j} c^{j l}$, where $c:=e^{\frac{2 \pi i}{N}}$. Also the $\boldsymbol{Q}_{k}$ 's will be chosen in an appropriate way such that, as in (Hwang et al. 1995) for the CMPP, the approximating process will be completely determined by two vectors, namely $\boldsymbol{a}$, the first row of $\boldsymbol{Q}$ and a rate vector $\boldsymbol{\gamma} . \boldsymbol{Q}_{\boldsymbol{k}}$ will be chosen here as $\left(\boldsymbol{Q}_{k}\right):=a_{(j-i) \bmod N} \frac{\left(\gamma_{i}\right)^{k} e^{-\gamma_{i}}}{k !}$. However, the $\boldsymbol{Q}_{k}$ 's may also be chosen differently. As long as $\sum_{k=0}^{\infty} k Q_{k}=a^{T} \gamma$, everything what follows will stay valid. Thus, it is also possible to define only a finite number of $\boldsymbol{Q}_{\boldsymbol{k}}$ 's different from the zero matrix. In any case, the input rate vector $\Gamma$ will then equal the rate vector $\boldsymbol{\gamma}$.

To find $a$, a set of linear programming problems equivalent with those described in (Hwang et al. 1995) have to be solved until a solution is found. The only difference is that there ae needs to be zero (since a CMPP is a continuous-time Markov chain) while here it needs to be 1 , with all $a_{j} \geq 0$ for $0 \leq j \leq N-1$. In our implementation of this solution method, the faster index search algorithm (ISA) as proposed in (Che et al. 1997) is used instead of the ad-hoc scheme developed in (Hwang et al. 1995).

An extension to periodic transition matrices is made in the following way: if $\boldsymbol{Q}$ needs to have a period $d>1$, it suffices to search only for eigenvalues with an argument in $\left[0, \frac{2 \pi}{d}[\right.$, because the set of eigenvalues of a periodic irreducible matrix, regarded as a system of points in the complex plane, is invariant under a rotation of the plane by the angle $\frac{2 \pi}{d}$ (see (Cinlar 1975)). By imposing that all the $a_{j}$ 's, with $j \neq k d+1$, for some $k \in \mathbb{N}$ are zero, also the eigenvalues with argument outside $\left[0, \frac{2 \pi}{d}\right.$ [ will automatically be eigenvalues of $\boldsymbol{Q}$.

Once the solution $\boldsymbol{a}$ is found, the contribution $\left(\psi_{c}\right)_{l}$ of each eigenvalue $\left(\lambda_{c}\right)_{l}$ to the power spectrum $P_{c}(\omega)$ of the approximation has to be found. For a circulant D-BMAP, $\left(\psi_{c}\right)_{l}=\frac{1}{N^{2}}\left(\sum_{j=0}^{N-1} \gamma_{j} c^{-l j}\right)\left(\sum_{j=0}^{N-1} \gamma_{j} c^{l j}\right)\left(\lambda_{c}\right)_{l}$, which implies that $\left(\psi_{c}\right)_{l}$ is a positive real multiple of $\left(\lambda_{c}\right)_{l}$. By defining $\beta_{k}$ as $\beta_{k}=\frac{1}{N} \sum_{l=0}^{N-1} \gamma_{l} c^{l k}$, and transforming it to polar notation, $\beta_{k}=\sqrt{\chi_{k}} e^{i \alpha_{k}}$, $\left(\psi_{c}\right)_{l}$ can be written as $\left(\psi_{c}\right)_{l}=\chi_{l}\left(\lambda_{c}\right)_{l}$. So, the problem is reduced to finding positive real $\chi_{l}$ 's. Of course the constructed $Q$ has also other eigenvalues besides the envisaged eigenvalues. To eliminate their contribution to $P_{c}(\omega)$, the corresponding $\chi_{l}$ 's are chosen zero. The $\chi_{l}$ 's corresponding with eigenvalues of modulus one can be calculated exactly such that the discrete part of the power spectrum which is introduced by those eigenvalues is matched exactly: if $\left(\lambda_{c}\right)_{0}=1$ then $\chi_{0}:=\left(\sum_{i=1}^{M} \sqrt{\psi_{0}^{(i)}}\right)^{2}$, if $\left(\lambda_{c}\right)_{a}=-1$ then $\chi_{a}:=-\sum_{i=1}^{M} \psi_{a}^{(i)}$ and if $\left(\lambda_{c}\right)_{l} \in \mathbb{C} \backslash \mathbb{R},\left|\left(\lambda_{c}\right)_{l}\right|=1$ then $\chi_{l}:=\sum_{i=1}^{M}\left|\psi_{l}^{(i)}\right|$. The other $\psi_{l}$ are 
found by minimising the difference between the continuous part of the power spectrum of the circulant D-BMAP and of the superposition by using the nonnegative least square method as in (Hwang et al. 1995).

At this point, the transition matrix $\boldsymbol{Q}$ of the circulant D-BMAP and the corresponding values $\left(\psi_{c}\right)_{l}$ are determined such that $P(\omega) \approx P_{c}(\omega)$. Now, only $\boldsymbol{\gamma}$ has still to be found such that $\left(\boldsymbol{Q}_{k}\right)_{k \geq 0}$ is completely defined. Of course, $\boldsymbol{\gamma}$ has to be determined in such a way that the $\left(\psi_{c}\right)_{l}$ 's, which depend on $\gamma$, are not changed anymore. The components of $\boldsymbol{\gamma}$ can be expressed as function of the $\chi_{l}$ 's and the $\alpha_{l}$ 's: if $N$ is odd $(N=2 p+1)$,

$$
\begin{aligned}
& \gamma_{t}=\sqrt{\chi_{0}}+2 \sum_{m=1}^{p} \sqrt{\chi_{m}} \cos \left(\alpha_{m}-\frac{2 \pi}{N} t m\right), \quad \text { or } \\
& \gamma_{t}=\sqrt{\chi_{0}}+2 \sum_{m=1}^{p-1} \sqrt{\chi_{m}} \cos \left(\alpha_{m}-\frac{2 \pi}{N} t m\right)+\sqrt{\chi_{p}} \cos \left(\alpha_{p}-\frac{2 \pi}{N} t p\right)
\end{aligned}
$$

if $N$ is even $(N=2 p)$. Remark that the value of $N$ will depend on the outcome of the index search algorithm. The only fact known about $N$ is that it has to be a multiple of the period.

As can be seen from (9) or (10), there is still a degree of freedom left, namely the $\alpha_{l}$ 's. Those will be used to match the stationary cumulative distribution of the input rate process. The stationary cumulative distribution $F(x)$ of $\Gamma$, the input rate in a slot of a D-BMAP $\left(\boldsymbol{D}_{k}\right)_{k \geq 0}$, is defined as $F(x)=P\{\Gamma \leq x\}$. Since $P\left\{\Gamma=\Gamma_{i}\right\}=\pi_{i}, F(x)$ is completely determined by $\pi$, the stationary distribution of $D$ and the input rate vector $\Gamma=\left(\Gamma_{0} \ldots \Gamma_{N-1}\right)^{T}=\sum_{k=0}^{\infty} k D_{k} e: F(x)=\sum_{\Gamma_{i} \leq x} \pi_{i}$. For the superposition of $M$ independent D-BMAPs $\left(D_{k}^{(i)}\right)_{k \geq 0}, \Gamma=\bigoplus_{i=1}^{M} \Gamma^{(i)}$ and $\pi=\bigotimes_{i=1}^{M} \pi^{(i)}$ such that $F(x)$, the stationary cumulative distribution of the aggregate input rate process is completely known from the cumulative distribution of the individual D-BMAPs. In order to match $F(x)$ and $F_{c}(x)$, which must be a cumulative distribution function that jumps by $\frac{1}{N}$ at each value $x \in \gamma$ (since the stationary distribution of a circulant is $\pi_{c}=\frac{1}{N} e^{T}$ ), the range of $x$ is partitioned into a set of $N$ equal probability rates. The matching problem is then again identical to the matching of the rate distribution for a CMPP in (Hwang et al. 1995), which is solved as a minimisation problem, in which the components of $\boldsymbol{\alpha}$ are the parameters that can be tuned, and that is solved by using the Nelder Meade simplex search method (Nelder et al. 1965).

A problem which can pop up by implementing the method as described above is the calculation of the powerspectrum of a D-BMAP $\left(D_{k}\right)_{k \geq 0}$. As can be seen from (5), the complete eigenstructure (eigenvalues $\lambda_{l}+$ corresponding right and left eigenvectors $\boldsymbol{g}_{\boldsymbol{l}}$ and $\boldsymbol{h}_{\boldsymbol{l}}$ such that $\boldsymbol{h}_{\boldsymbol{l}} \boldsymbol{g}_{\boldsymbol{l}}=1$ ) of $\boldsymbol{D}$ needs to be known. Calculating the eigenvectors can for relatively large periodic matrices give unsatisfactory results. In that case, it is also possible to calculate the powerspectrum directly from the autocorrelation sequence by using the fact 
that appropriate subsequences of the autocorrelation sequence converge to limits which are known in closed form:

Property. If $\left(D_{k}\right)_{k \geq 0}$ is a D-BMAP of period d, and $\pi_{i},\left(\sum_{k=0}^{\infty} k D_{k}\right)_{i}$ and $\boldsymbol{e}_{i}$ are parts of $\pi, \sum_{k=0}^{\infty} k D_{k}$ and $e$ corresponding to a permutation of the states of a periodic $\boldsymbol{D}$ which can always be made (Çinlar 1975), then

$$
\begin{array}{r}
R[m d+l] \stackrel{m \rightarrow \infty}{\longrightarrow} l i m_{l}=d \sum_{i=0}^{d-1} \pi_{i}\left(\sum_{k=0}^{\infty} k D_{k}\right)_{i} e_{(i+1) \bmod d} \\
\pi_{(i+l) \bmod d}\left(\sum_{k=0}^{\infty} k D_{k}\right)_{(i+l) \bmod d} \boldsymbol{e}_{(i+l+1) \bmod d}
\end{array}
$$

This property is proven in an analog way as the proof in (Geerts 1997) of the property that appropriate subsequences of the covariance sequence of the number of arrivals in a time slot of a periodic D-MAP (this is a D-BMAP with $\boldsymbol{D}_{k}=\boldsymbol{O}$ if $\left.k \geq 2\right)$ converge. By replacing $R[d k+l]$ by its limit as soon as $\left|R[d k+l]-\lim _{l}\right|<\epsilon$, an approximation for $P(\omega)$ can be calculated directly from the autocorrelation sequence.

\section{APPLICATION OF THE CIRCULANT MATCHING METHOD TO VIDEO SOURCES}

The circulant matching method for D-BMAPs of subsection 2.3 needs now of course some validation. For this, the method is applied to an MPEG source type model developed in (Helvic 1996). This model was used in a series of Connection Admission Control (CAC) experiments (Aarstad et al. 1998) performed in the EXPERT project of the European Telecommunications research programme ACTS at the ATM testbed in Basle, Switzerland.

In MPEG encoding of a video sequence, three different compression levels are used. Thereby, three types of frames, namely I-, P- and B-frames are generated. After encoding, the frames are arranged in a periodic deterministic sequence (group of pictures) usually of length 12 in the pattern IBBPBBPBBPBB.

In (Helvic 1996) and (Conti et al. 1996), a Markov model for an MPEG source is presented. The model used here is the one of (Helvic 1996). It is level oriented (see Figure 1), where level $i$ models the activity of the MPEG source when the generated load of the B- and P-frames between two I-frames is between $l_{i}$ and $l_{i+1}$. Within a level, the B- and $\mathrm{P}$-frame activities are modelled by single states, the I-frame load by an approximate distribution given the level. Parameters in the model are the transition probabilities between the different states of the model and a load for each state (expressed in bits per frame) which is generated while being in that state. The state sojourntime is $45 \mathrm{msec}$ for all states (a frame duration). 
Two examples of MPEG sources are used in the experiments and also modelled as D-BMAPs. They are based on a 24 minutes trace from the Bond movie "Goldfinger" and a 24 minutes trace from an Asterix cartoon. Both traces have been made available by O.Rose at the Institute of Computer Science at the University of Würzburg. The source based on the Bond movie is modelled with 5 load levels and two I-frames at each level (and thus 65 states) while the Asterix cartoon is modelled with 4 load levels and also $2 \mathrm{I}$-frames at each level (52 states). In the sequel of this paper, these sources will be refered to as "Bond" and "Asterix".

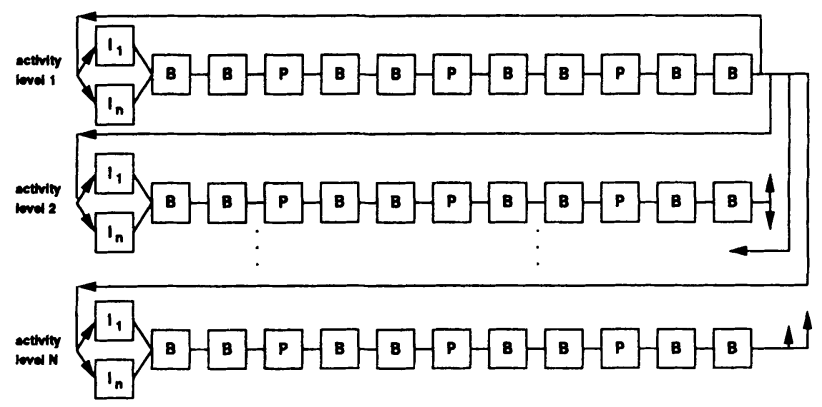

Figure 1 Structure of the artificial Markovian MPEG source

A D-BMAP $\left(D_{k}\right)_{k \geq 0}$ can be constructed from the data generated by the method of (Helvic 1996) by using the given transition matrix as the transition matrix $\boldsymbol{D}$ for the D-BMAP and by transforming the load given for each state into number of cells per frame. If this results in $n$ cells per frame for state $i$, define $\forall j:\left(\boldsymbol{D}_{n}\right)_{i, j}:=(\boldsymbol{D})_{i, j},\left(\boldsymbol{D}_{m}\right)_{i, j}:=0, \forall m \neq n$.

Application of the circulant matching method to D-BMAPs based on the MPEG model for the superposition of Bond sources, Asterix sources or a combination of those two, gives a new D-BMAP $(\boldsymbol{a}, \boldsymbol{\gamma})$ which is an approximation for the superposition. Since $\boldsymbol{D}$ has periodicity 12 (see Figure 1) and dimension 52 for the Asterix source, a search for a circulant $Q$ which has as required eigenvalues the 4 eigenvalues of $D$ which are different from zero and have their argument in $\left[0, \frac{\pi}{6}\right.$ [ is performed. By imposing $\boldsymbol{Q}$ to have also period 12 , as described in section $2.3, Q$ will have as eigenvalues among others all the eigenvalues of $D$. The result is a circulant of dimension 132. For the Bond source, $\boldsymbol{D}$ has 5 eigenvalues in the segment $\left[0, \frac{\pi}{6}\right.$ [ and the dimension of the resulting circulant is also 132. For the combination of Bond and Asterix there are 8 eigenvalues with argument in $\left[0, \frac{\pi}{6}[: 4\right.$ come from the Asterix source and 5 from the Bond source, but of course they have $\lambda_{0}=1$ in common. The result is a circulant of dimension 276 . These dimensions will stay the same irrespective of the number of sources that is multiplexed. The difference will be in $\gamma$. For Bond and Asterix sources this means thus that as soon as two sources are multiplexed, the dimensions of the approximation are smaller than those of the exact superposition. 
The underlying time unit for the circulant D-BMAPs $(a, \gamma)$ is still a frame length of $45 \mathrm{msec}$. Since this D-BMAP will be used as input for a single server queue with a constant service time which equals the time needed to transmit one cell onto the outgoing link ( $=1$ slot), $(a, \gamma)$ has to be transformed into a D-BMAP $(\hat{\boldsymbol{a}}, \hat{\gamma})$ with one slot as the underlying time unit. If it is supposed that the number of slots of being in a state is geometrically distributed with mean $x$, where $x$ is the number of slots in a frame duration, $p=1-\frac{1}{x}$ is the probability to go from a state to the same state after one slot. The D-BMAP $(\boldsymbol{a}, \boldsymbol{\gamma})$ can thus be transformed to $(\hat{\boldsymbol{a}}, \hat{\boldsymbol{\gamma}})$ by replacing $a_{0}$ by $p\left(a_{0}\right.$ is 0 since $\boldsymbol{Q}$ is periodic) and multiplying all the other elements of $a$ by $(1-p)$. Further, all the elements of $\gamma$ have to be divided by $x$.

\section{RESULTS}

Experimental multiplexing results were obtained in the EXPERT ATM testbed by using a traffic generator and analyser instrument called ATM-100 which gives the possibility to generate and analyse quite general random traffic. The ATM-100 is equipped with two Synthesised Traffic Generators (STGs) that are used for generating the artificial MPEG traffic. The traffic is multiplexed on an output port of a Fore ASX-200 switch with a buffer of 100 cells. Due to hardware constraints in the traffic generators a pacing rate function has been used to limit the output port capacity to $37.44 \mathrm{Mbit} / \mathrm{sec}$, thereby reducing the number of sources required to adequately load the system. The aggregate traffic stream is analysed in the ATM-100, permitting cell loss measurements.

Those multiplexing experiments were performed in the framework of a CAC investigation for video and data and are reported in detail in (Aarstad et al. 1998). The experiments are based on multiplexing, since the way to perform them is to change the traffic mix until a CLR below, but as close as possible to a fixed value is obtained. These experimental multiplexing results are used to validate the circulant matching method for D-BMAPs.

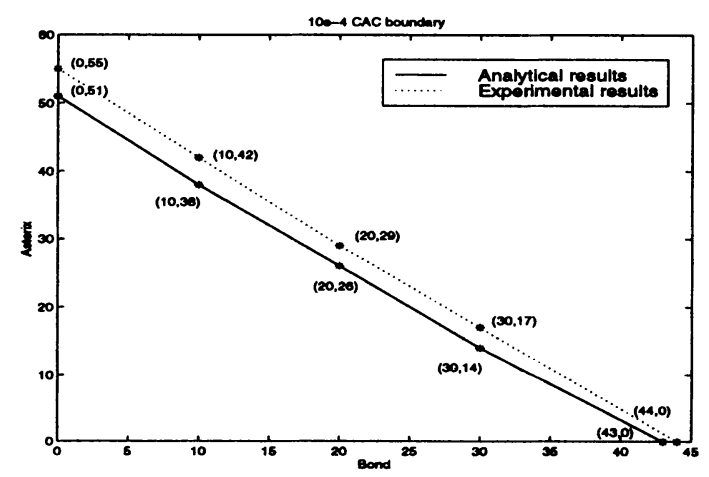

Figure 2 Comparisson of analytically and experimentally obtained results 
To be able to compare results with the experimental results, the circulants obtained for multiplexing a number of Bond and Asterix sources have to be fed into a multiplexer and values for the CLR have to be calculated. This multiplexer is modelled as a discrete-time $\mathrm{D}-\mathrm{BMAP} / \mathrm{D} / 1 / \mathrm{K}+1$ queueing system, where $K$ is the buffer size and $K+1$ the system size. The service time equals one time slot, i.e. the time needed to transmit one cell on the outgoing link. In (Blondia et al. 1992), the D-BMAP/D/1/K+1 model is solved and a formula for calculating the CLR is derived.

All the results presented are obtained for a queue length of 100 cells and an outgoing link of capacity $37.44 \mathrm{Mbit} / \mathrm{sec}$. This implies that one time slot equals $11.325 \mu \mathrm{sec}$. By transforming bits into number of cells, it is assumed that a cell of 53 bytes can contain 48 bytes of data. Figure 2 compares the analytically derived $10^{-4} \mathrm{CAC}$ boundary with the experimentally obtained one. As in the experiments, for a mix of two types of sources, the admission boundary is close to linear (Aarstad et al. 1998). If the points in Figure 2 are compared, it is seen that the analytical results are more conservative than the experimental results, with a larger deviation if the number of Asterix sources grows. For the D-BMAPs of the MPEG sources, the parameters as obtained from the method in (Helvic 1996) are used, which give rise to a mean arrival rate of 58.1212 cells $/ 45 \mathrm{msec}$ or $0.54820 \mathrm{Mbit} / \mathrm{sec}$ for the Asterix source and 63.3247 cells $/ 45 \mathrm{msec}$ or $0.59666 \mathrm{Mbit} / \mathrm{sec}$ for the Bond source. However, if the sources are implemented in the traffic generator, the parameters are automatically slightly changed to adapt them to the hardware limitations of the STGs. Depending on the number of sources generated, these slight differences may become more important. The first limitation is that the STGs can only provide transition probability values in integer multiples of $\frac{1}{256}$. The second limitation is that the peak rate in a state must divide the link rate such that the interarrival time between cells in a given state is always the same integer number of slots. The result is that the mean arrival rate for an experimental Asterix source is $0.51318 \mathrm{Mbit} / \mathrm{sec}$ and $0.59221 \mathrm{Mbit} / \mathrm{sec}$ for a Bond source. The analytical model for the Asterix source generates thus $0.03502 \mathrm{Mbit} / \mathrm{sec}$ more than the experimental model, which means that for a certain experimental point the corresponding analytical CLR will be worse depending on the number of Asterix sources used. This explains partially why in the analytical curve the number of sources that can be accepted is smaller than in the experiments, with a larger difference if more Asterix sources are involved. Analogous observations are found by simulation (Aarstad et al. 1998).

\section{CONCLUSIONS}

In this paper, the circulant matching method to approximate the superposition of a number of discrete-time batch Markovian arrival processes by matching the stationary cumulative distribution and the autocorrelation sequence of the input rate process was proposed. The method was applied to the super- 
position of MPEG source type models. For validation of the method, experimental results of experiments performed within the ACTS project EXPERT were used. The results confirm the accuracy of the method.

Acknowledgement. We would like to thank the partners of WP4.2 of the EXPERT project together with whom the experiments were performed. Also the valuable e-mail discussions with Hao Che are gratefully appreciated.

\section{REFERENCES}

Aarstad, E., S. Blaabjerg, F. Cerdan, S. Peeters and K. Spaey (1998) CAC investigation for video and data. To appear in Proceedings of IFIP TC 6 Fourth International Conference on Broadband Communications.

Blondia, C. and O. Casals (1992) Statistical Multiplexing of VBR Sources: A Matrix-Analytic Approach. Performance Evaluation, 16, 5-20.

Blondia, C. (1993) A Discrete-Time Batch Markovian Arrival Process as BISDN Traffic Model. Belgian Journal of Operations Research, Statistics and Computer Science, 32(3,4), 3-23.

Blondia, C. and F. Panken (1995) Traffic profile of a Connection in an ATM Network with Application to Traffic Control. Proceedings of ATM hot topics on Traffic and Performance, From Race to ACTS, Milan.

Che, H. and S.Q. Li (1997) Fast algorithms for Measurement-Based Traffic Modeling. Proceedings of IEEE INFOCOM '97.

Çinlar, E. (1975) Introduction to Stochastic Processes. Prentice-Hall, Englewood Cliffs, New Jersey.

Conti, M., E. Gregori and A. Larsson (1996) Study of the Impact of MPEG-1 Correlations on Video-Sources Statistical Multiplexing. IEEE Journal on Selected Areas in Communications, 14(7), 1455-71.

Geerts, F. (1997) A proof of the correlation decay of a periodic D-MAP. Available at http://win-www.uia.ac.be/u/fgeerts.

Helvic, B.E. (1996) MPEG source type models for the STG (Synthesized Traffic Generator). SINTEF Report STF40 A96016.

Hwang, C.L. and S.Q. Li (1995) On the Convergence of Traffic Measurement and Queueing Analysis: A Statistical MAtch Queueuing (SMAQ) Tool. Proceedings of IEEE INFOCOM '95, 602-12.

Landry, R. and I. Stavrakakis (1997) Multiplexing ATM Traffic Streams with Time-Scale-Dependent Arrival Processes. Computer Networks and ISDN Systems, 29.

Lazar, A., G. Pacifici and E. Pendarakis (1993) Modeling Video Sources for Real-Time Scheduling. IEEE GLOBECOM '93, Houston, Texas.

Li, S.Q. and C.L. Hwang (1992) Queue Response to Input Correlation Functions: Discrete Spectral Analysis. Proceedings of IEEE INFOCOM '92.

Nelder, J.A. and Mead, R. (1965) A Simplex Method for Function Minimization. Computer Journal, 7, 308-13. 